\title{
Corticosteroid use during pregnancy and risk of orofacial clefts
}

\author{
Anders Hviid MSc DMSc, Ditte Mølgaard-Nielsen MSc
}

Competing interests: None declared.

This article has been peer reviewed.

Correspondence to: Anders Hviid, aii@ssi.dk

CMAJ 2011. DOI:10.1503 /cmaj.101063

\section{ABSTRACT}

Background: The association between the risk of orofacial clefts in infants and the use of corticosteroids during pregnancy is unclear from the available evidence. We conducted a nationwide cohort study of all live births in Denmark over a 12-year period.

Methods: We collected data on all live births in Denmark from Jan. 1, 1996, to Sept. 30, 2008. We included live births for which information was available from nationwide health registries on the use of corticosteroids during pregnancy, the diagnosis of an orofacial cleft and possible confounders.

Results: There were 832636 live births during the study period. Exposure to corticosteroids during the first trimester occurred in 51973 of the pregnancies. A total of 1232 isolated orofacial clefts (i.e., cleft lip, cleft palate, or cleft lip and cleft palate) were diagnosed within the first year of life, including 84 instances in which the infant had been exposed to corticosteroids during the first trimester of pregnancy. We did not identify any statistically significant increased risk of orofacial clefts associated with the use of corticosteroids: cleft lip with or without cleft palate, prevalence odds ratio (OR) 1.05 (95\% confidence interval [Cl] 0.80-1.38]; cleft palate alone, prevalence OR 1.23 (95\% Cl 0.83-1.82). Odds ratios for risk of orofacial clefts by method of delivery (i.e., oral, inhalant, nasal spray, or dermatologic and other topicals) were consistent with the overall results of the study and did not display significant heterogeneity, although the OR for cleft lip with or without cleft palate associated with the use of dermatologic corticosteroids was 1.45 (95\% Cl $1.03-$ 2.05).

Interpretation: Our results add to the safety information on a class of drugs commonly used during pregnancy. Our study did not show an increased risk of orofacial clefts with the use of corticosteroids during pregnancy. Indepth investigation of the pattern of association between orofacial clefts and the use of dermatologic corticosteroids during pregnancy indicated that this result did not signify a causal connection and likely arose from multiple statistical comparisons.
$\mathrm{T}$ he anti-inflammatory and immunosuppressive properties of corticosteroids in pharmacotherapeutic doses has a wide range of clinical uses, such as for the treatment of asthma, atopic dermatitis and other allergic conditions, autoimmune diseases and cancer. However, caution is warranted for the use of corticosteroid medications during pregnancy. Corticosteroid use during pregnancy has been associated with orofacial clefts in animals, and similar risks in humans are suspected. ${ }^{1,2}$ The available epidemiologic evidence favours an association, but many of the studies that have been done have been limited by recall bias and a lack of statistical power. The association between risk of orofacial clefts and the use of corticosteroids during pregnancy remains unclear. ${ }^{3-10}$

We conducted a nationwide cohort study in Denmark with independent and prospective determination of corticosteroid use during pregnancy and the diagnosis of orofacial clefts. Our study comprised all live births from January 1996 to September 2008.

\section{Methods}

\section{Study cohort}

The Danish Medical Birth Registry was established in 1968 and contains information on all births in Denmark. ${ }^{11}$ Each record in the registry includes the following information: the personal identification number (a 10-digit number assigned to all people living in Denmark and used in all nationwide registries) of the mother, the father (when known) and the newborn (for all live births); the date and time of birth; any complications during pregnancy or delivery; and the gestational age, birth weight and other physical characteristics of the newborn, including any malformations diagnosed at birth. Estimation of gestational age is primarily done by ultrasound. ${ }^{12}$ However, the date of the last menstrual period is sometimes used. 
Using the information in the medical birth registry, we constructed a cohort of all live births in Denmark from Jan. 1, 1996, to Sept. 30, 2008. The date on which each pregnancy began was estimated by subtracting the estimated gestational age from the date of birth. Births with missing gestational age (7371 births [0.9\%]) were excluded, and the final cohort included 832636 births.

\section{Use of corticosteroids during pregnancy}

Information on all corticosteroid prescriptions given to women in the cohort and filled during the period starting four weeks before pregnancy and ending at birth was obtained from the Danish Prescription Drug Register. This register contains information on all prescriptions filled at Danish pharmacies, the only places in Denmark where prescription drugs can legally be purchased, since 1995. Each record is indexed using the recipient's personal identification number and includes the date on which the prescription was filled, the Anatomic Therapeutic Chemical (ATC) code of the drug (available from www.whocc.no/atc_ddd _index/), the number of packages dispensed, the size of the package dispensed and the number of daily defined doses in the prescription. For the purposes of our study, the date on which the prescription was filled was considered the date of use. We included corticosteroid drugs with the following ATC codes in the study: A01AC, A07EA, C05AA, D07, D10AA, G01B, H02A, H02B, M01BA, N02CB, R01AD, R03BA, S01BA, S01BB, S01CA, S01CB, S02B, S02C, S03B and S03C. We grouped corticosteroid drugs according to the route of administration (oral, inhalant, nasal spray, and dermatologic and other topicals).

\section{Orofacial clefts and other diagnostic information}

Infants with orofacial clefts (clefts) were identified through the National Hospital Discharge Register. ${ }^{13}$ This registry was established in 1977 and contains information on hospital contacts indexed by the personal identification number. The registry includes information on the dates of admission and discharge as well as the diagnoses at discharge. We had access to data for the period covering Jan. 1, 1996, to Mar. 31, 2009. The 10th revision of the International Statistical Classification of Diseases and Related Health Problems (ICD-10) was used to code diagnostic information. Clefts were subcategorized as cleft lip with or without cleft palate (ICD-10 codes Q36 and Q37) and cleft palate alone (ICD-10 code Q35). Only diagnoses made during the first year of life were included.
Clefts were further characterized as isolated or as one of multiple birth defects (any diagnosis of a birth defect other than clefts, ICD-10 codes Q00-Q89, during the first year of life) using information from the discharge registry. Orofacial clefts in isolation were used as the main outcome measure.

We excluded children with chromosomal abnormalities (ICD-10 codes Q90-Q99) diagnosed during the first year of life from the cohort.

\section{Potential confounders}

We included information on many potential confounders. These confounders were selected using previously described associations with clefts found in the literature. ${ }^{1423}$

Information on maternal age at the start of pregnancy, parity and smoking status during pregnancy was obtained from the Danish Medical Birth Registry; the mother's place of origin and her place of residence at the start of pregnancy were obtained from the Danish Central Person Register, which contains complete and continually updated demographic information on all Danish residents; ${ }^{24}$ the level of education and socioeconomic status of the mother during the year in which pregnancy started was obtained from Statistics Denmark.

Information on the following maternal morbidities was obtained from the discharge registry: infections during the first trimester (ICD-10 codes A00-B99), infections of the genitourinary tract during the first trimester (ICD-10 code O23), diabetes (ICD-10 codes O24 and E10E14) and epilepsy (ICD-10 codes G40 and G41).

Information on the use of the following additional drugs during the first trimester was obtained from the Danish Prescription Drug Register: antiepileptic medications (ATC code N03), benzodiazepines (ATC code N05BA), $\beta$-blockers (ATC code C07A), oral contraceptives (ATC code G03A) and analgesic agents (ATC code N02).

The history of birth defects among siblings dating from the end of the study period back to 1977 was obtained from the discharge registry.

Finally, the year in which pregnancy began was also included in our analysis.

\section{Statistical analysis}

We used logistic regression analysis (SAS procedure PROC GENMOD) to estimate prevalence odds ratios (ORs) with 95\% confidence intervals (CIs) to compare the prevalence odds of clefts among births with and without exposure to corticosteroids during pregnancy. In our main analysis, we estimated the effects of using corticosteroids during the first trimester (defined as the first 12 
weeks after the start of pregnancy), the period including primary organogenesis during which exposure is most likely to cause structural defects.

Covariables that were potential confounders were included in the regression models if they were significant (likelihood ratio test $p<0.05$ ) risk factors for clefts in univariable analyses. In univariable analyses, $p$ values were estimated with missing values excluded. The covariables were evaluated separately for cleft lip with or without cleft palate and cleft palate alone, yielding two sets of variables included in all further regression models of the two outcomes. Since the proportions of missing values were all less than $5 \%$, we used mode imputation for missing values in adjusted models.

Table 1: Frequency distributions of exposure to corticosteroids among 832636 live births in Denmark (part 1 of 2)

\begin{tabular}{|c|c|c|c|c|c|}
\hline \multirow[b]{2}{*}{ Characteristic } & \multicolumn{3}{|c|}{ No. (\%) exposed } & \multirow[b]{2}{*}{$\begin{array}{l}\text { No. }(\%) \text { not } \\
\text { exposed } \\
n=669142\end{array}$} & \multirow[b]{2}{*}{ Total no. (\%) } \\
\hline & $\begin{array}{c}1-4 \text { wk before } \\
\text { pregnancy } \\
n=21859\end{array}$ & $\begin{array}{l}\text { First trimester } \\
\quad n=51973\end{array}$ & $\begin{array}{c}\text { Second and } \\
\text { third trimesters } \\
n=121690\end{array}$ & & \\
\hline \multicolumn{6}{|l|}{ Year of birth } \\
\hline 1996-1998 & $5474 \quad(25.0)$ & 12400 & $26894(22.1)$ & 162483 & $199863(24.0)$ \\
\hline 1999-2001 & $5257 \quad(24.1)$ & 12508 (24.1) & 28419 (23.4) & 159424 (23.8) & $197823(23.8)$ \\
\hline 2002-2004 & $5039 \quad(23.1)$ & $11943(23.0)$ & $28234(23.2)$ & $154843 \quad(23.1)$ & $192674(23.1)$ \\
\hline $2005-2008$ & 6089 (27.9) & $15122(29.1)$ & 38143 (31.3) & $192392(28.8)$ & $242276(29.1)$ \\
\hline \multicolumn{6}{|c|}{ Maternal age at start of pregnancy, yr } \\
\hline$<18$ & $49 \quad(0.2)$ & $104 \quad(0.2)$ & $186 \quad(0.2)$ & $1944 \quad(0.3)$ & $2232 \quad(0.3)$ \\
\hline $18-24$ & 2567 (11.7) & 6127 (11.8) & $12670(10.4)$ & $93620(14.0)$ & $111814(13.4)$ \\
\hline $25-29$ & 7399 (33.9) & 17882 & 39959 (32.8) & 235779 (35.2) & 290506 (34.9) \\
\hline $30-34$ & $8062 \quad(36.9)$ & 18805 (36.2) & $45840 \quad(37.7)$ & 233550 & 294103 (35.3) \\
\hline $35-39$ & 3228 (14.8) & 7729 (14.9) & 19818 (16.3) & $90051 \quad(13.5)$ & 115617 (13.9) \\
\hline $40-44$ & $541 \quad(2.5)$ & 1284 & 3113 & 13755 & $17787 \quad(2.1)$ \\
\hline$\geq 45$ & $13 \quad(0.1)$ & $42 \quad(0.1)$ & $104 \quad(0.1)$ & $443 \quad(0.1)$ & $577 \quad(0.1)$ \\
\hline \multicolumn{6}{|l|}{ Maternal parity } \\
\hline 0 & $8655 \quad$ (39.6) & 21308 (41.0) & 48906 (40.2) & 288720 & 354451 (42.6) \\
\hline 1 & $8272 \quad$ (37.8) & 19299 (37.1) & 46192 (38.0) & $239526 \quad(35.8)$ & 301448 (36.2) \\
\hline 2 & 3138 (14.4) & 7373 (14.2) & 17683 (14.5) & 91970 (13.7) & 115647 (13.9) \\
\hline$\geq 3$ & $1311 \quad(6.0)$ & $2906 \quad(5.6)$ & $6623 \quad(5.4)$ & 34281 & $43208 \quad$ (5.2) \\
\hline Unknown & $483 \quad(2.2)$ & 1087 & 2286 & $14645 \quad(2.2)$ & $17882 \quad(2.2)$ \\
\hline \multicolumn{6}{|c|}{$\begin{array}{l}\text { Maternal place of residence at start } \\
\text { of pregnancy }\end{array}$} \\
\hline Capital region & $7023 \quad(32.1)$ & 16609 (32.0) & 39430 (32.4) & 215766 & 268558 (32.3) \\
\hline Sealand & $2805 \quad(12.8)$ & $6470 \quad(12.5)$ & 15197 (12.5) & $85641 \quad(12.8)$ & 106128 (12.8) \\
\hline Southern Denmark & $4764 \quad(21.8)$ & 10828 & 24858 (20.4) & 138892 & 172595 (20.7) \\
\hline Middle Jutland & $5140 \quad(23.5)$ & 12693 & 29206 (24.0) & $151616 \quad(22.7)$ & 190832 (22.9) \\
\hline Northern Jutland & $2084 \quad(9.5)$ & 5228 (10.1) & $12075 \quad(9.9)$ & 66495 & $82736 \quad(9.9)$ \\
\hline Unknown & $43 \quad(0.2)$ & $145 \quad(0.3)$ & $924 \quad(0.8)$ & 10732 & 11787 \\
\hline \multicolumn{6}{|l|}{ Maternal place of origin } \\
\hline Denmark & 18764 (85.8) & 44414 (85.5) & $105673(86.8)$ & $572403 \quad(85.5)$ & 713522 (85.7) \\
\hline Europe or North America & $886 \quad(4.1)$ & $2332 \quad(4.5)$ & 5573 & 34463 & $41858 \quad(5.0)$ \\
\hline Other & 2110 & $5036 \quad(9.7)$ & $10047 \quad(8.3)$ & 59472 & $73876 \quad$ (8.9) \\
\hline Unknown & $(0.5)$ & $191 \quad(0.4)$ & $397 \quad(0.3)$ & 2804 & $3380 \quad(0.4)$ \\
\hline \multicolumn{6}{|l|}{ Maternal level of education } \\
\hline Compulsory school & $4801 \quad(22.0)$ & 11179 (21.5) & $24386(20.0)$ & 143542 & 177363 (21.3) \\
\hline Secondary school & $2747 \quad(12.6)$ & $6510 \quad(12.5)$ & $14954(12.3)$ & $87367 \quad(13.1)$ & 107553 (12.9) \\
\hline $\begin{array}{l}\text { Vocational training or some } \\
\text { postsecondary education }\end{array}$ & $7941 \quad(36.3)$ & 19007 (36.6) & 44853 (36.9) & $234366 \quad(35.0)$ & 294629 (35.4) \\
\hline
\end{tabular}




\section{Results}

A total of 832636 live births with known gestational age and no chromosomal abnormalities were included in the study cohort. Of these, $798003(95.8 \%)$ were singleton births and 34633 (4.2\%) were multiple births.

We identified 1232 isolated orofacial clefts diagnosed during the first year of life (305 cleft lips, 570 cleft lips with cleft palates and 357 cleft palates alone). The corresponding rates of isolated clefts diagnosed during the first year of life were $1.48,1.05$ and 0.43 per 1000 births.

In our cohort, 163494 women (19.6\%) used corticosteroids at least once during the period from four weeks before pregnancy to birth. Use

Table 1: Frequency distributions of exposure to corticosteroids among 832636 live births in Denmark (part 2 of 2)

\begin{tabular}{|c|c|c|c|c|c|}
\hline \multirow[b]{2}{*}{ Characteristic } & \multicolumn{3}{|c|}{ No. (\%) exposed } & \multirow[b]{2}{*}{$\begin{array}{l}\text { No. }(\%) \text { not } \\
\text { exposed } \\
n=669142\end{array}$} & \multirow[b]{2}{*}{ Total no. (\%) } \\
\hline & $\begin{array}{l}1-4 \text { wk before } \\
\text { pregnancy } \\
n=21859\end{array}$ & $\begin{array}{l}\text { First trimester } \\
n=51973\end{array}$ & $\begin{array}{c}\text { Second and } \\
\text { third trimesters } \\
n=121690\end{array}$ & & \\
\hline \multicolumn{6}{|l|}{$\begin{array}{l}\text { Maternal level of education } \\
\text { (continued) }\end{array}$} \\
\hline Postsecondary education & $5817(26.6)$ & $13830(26.6)$ & 33883 (27.8) & $175648(26.3)$ & 220046 (26.4) \\
\hline Unknown & $553 \quad(2.5)$ & 1447 & 3614 & $28219 \quad(4.2)$ & $33045 \quad(4.0)$ \\
\hline \multicolumn{6}{|l|}{ Maternal socioeconomic status } \\
\hline Unemployed & $5584(25.6)$ & $13220(25.4)$ & $28606(23.5)$ & $167977(25.1)$ & 207363 (24.9) \\
\hline $\begin{array}{l}\text { Employment with minimal } \\
\text { qualifications }\end{array}$ & $7640(35.0)$ & $18054(34.7)$ & 42031 (34.5) & 230945 (34.5) & 287883 (34.6) \\
\hline $\begin{array}{l}\text { Employment with midlevel } \\
\text { qualifications }\end{array}$ & $4422(20.3)$ & $10420(20.1)$ & $25396(20.9)$ & $130737(19.5)$ & 164146 (19.7) \\
\hline $\begin{array}{l}\text { Employment with unknown } \\
\text { qualifications }\end{array}$ & 1212 & $3040 \quad(5.9)$ & $7225 \quad(5.9)$ & $41693 \quad(6.2)$ & $51414 \quad(6.2)$ \\
\hline $\begin{array}{l}\text { Self-employed or employed by } \\
\text { spouse }\end{array}$ & $488 \quad(2.2)$ & 1219 & $3067 \quad(2.5)$ & $16387 \quad(2.5)$ & $20416 \quad$ (2.5) \\
\hline Managerial position & 2496 (11.4) & $5966(11.5)$ & $15127(12.4)$ & 78413 (11.7) & 98146 (11.8) \\
\hline Unknown & $17 \quad(0.1)$ & $54 \quad(0.1)$ & $238 \quad(0.2)$ & $2990 \quad(0.5)$ & $3268 \quad(0.4)$ \\
\hline \multicolumn{6}{|l|}{ Smoking status during pregnancy* } \\
\hline Did smoke & $4010(18.3)$ & $9454(18.2)$ & $23013(18.9)$ & $130607(19.5)$ & 161498 (19.4) \\
\hline Unknown & $832 \quad(3.8)$ & 1912 & 4137 & 23179 & 28855 \\
\hline $\begin{array}{l}\text { History of orofacial clefts among } \\
\text { offspring* }\end{array}$ & $48 \quad(0.2)$ & $83 \quad(0.2)$ & $180 \quad(0.2)$ & $1161 \quad(0.2)$ & $1422 \quad(0.2)$ \\
\hline $\begin{array}{l}\text { History of birth defects among } \\
\text { offspring* }\end{array}$ & 1134 & $2696 \quad(5.2)$ & $6347 \quad(5.2)$ & $31170 \quad(4.7)$ & $39652 \quad(4.8)$ \\
\hline \multicolumn{6}{|l|}{ Maternal diseases* } \\
\hline $\begin{array}{l}\text { Infectious disease during first } \\
\text { trimester }\end{array}$ & $72 \quad(0.3)$ & $198 \quad(0.4)$ & $382(0.3)$ & $1613 \quad(0.2)$ & $2134 \quad(0.3)$ \\
\hline $\begin{array}{l}\text { Infection of the genitourinary } \\
\text { tract during first trimester }\end{array}$ & $128 \quad(0.6)$ & $291 \quad(0.6)$ & $694 \quad(0.6)$ & $3092 \quad(0.5)$ & $4016 \quad(0.5)$ \\
\hline History of diabetes & $305 \quad(1.4)$ & $717 \quad(1.4)$ & 1540 & $7108 \quad(1.1)$ & $9193 \quad(1.1)$ \\
\hline History of epilepsy & $142 \quad(0.7)$ & $317 \quad(0.6)$ & $740 \quad(0.6)$ & $3663 \quad(0.5)$ & $4663 \quad(0.6)$ \\
\hline \multicolumn{6}{|l|}{$\begin{array}{l}\text { Maternal drug use during first } \\
\text { trimester* }\end{array}$} \\
\hline Antiepileptic agent & $82 \quad(0.4)$ & $213 \quad(0.4)$ & $458 \quad(0.4)$ & $2165 \quad(0.3)$ & $2779 \quad(0.3)$ \\
\hline Benzodiazepine & $137 \quad(0.6)$ & $333 \quad(0.6)$ & $671 \quad(0.6)$ & $2355 \quad(0.4)$ & $3245 \quad(0.4)$ \\
\hline$\beta$-Blocker & $98 \quad(0.5)$ & $243 \quad(0.5)$ & $561 \quad(0.5)$ & $2029 \quad(0.3)$ & $2741 \quad(0.3)$ \\
\hline Oral contraceptive & $221 \quad(1.0)$ & $708 \quad(1.4)$ & 1387 & $8012 \quad(1.2)$ & $9938 \quad(1.2)$ \\
\hline Analgesic agent & $410 \quad(1.9)$ & $988 \quad(1.9)$ & 1924 & $6251 \quad(0.9)$ & $8842 \quad(1.1)$ \\
\hline
\end{tabular}

*Numbers not shown for women who did not smoke during pregnancy, who did not have previous children with birth defects, who did not have the specified diseases or who did not use the specified drugs. 
Table 2: Risk factors associated with isolated orofacial clefts among 832636 live births in univariable analysis

\begin{tabular}{|c|c|c|c|c|}
\hline Risk factor & $N$ & $\begin{array}{c}n \\
\text { (prevalence } \\
\text { per } 1000 \text { births) }\end{array}$ & OR $(95 \% \mathrm{Cl})$ & $p$ value* \\
\hline \multicolumn{5}{|c|}{ Cleft lip with or without cleft palate } \\
\hline \multicolumn{5}{|l|}{ Year of birth } \\
\hline 1996-1998 & 199863 & $233(1.17)$ & 1.00 & \\
\hline 1999-2001 & 197823 & $218(1.10)$ & $0.95(0.79-1.14)$ & \\
\hline 2002-2004 & 192674 & $215(1.11)$ & $0.96(0.80-1.15)$ & \\
\hline 2005-2008 & 242276 & $209(0.09)$ & $0.74(0.61-0.89)$ & 0.006 \\
\hline \multicolumn{5}{|l|}{$\begin{array}{l}\text { Maternal residence at start } \\
\text { of pregnancyt }\end{array}$} \\
\hline Capital region & 268558 & $272(1.01)$ & 1.00 & \\
\hline Sealand & 106128 & $141(1.33)$ & $1.31(1.07-1.61)$ & \\
\hline Southern Denmark & 172595 & $181(1.05)$ & $1.04(0.86-1.25)$ & \\
\hline Middle Jutland & 190832 & $202(1.06)$ & $1.05(0.87-1.25)$ & \\
\hline Northern Jutland & 82736 & $71(0.86)$ & $0.85(0.65-1.10)$ & 0.030 \\
\hline \multicolumn{5}{|l|}{ Maternal place of origin $\ddagger$} \\
\hline Denmark & 713522 & 779 (1.09) & 1.00 & \\
\hline Europe and North America & 41858 & $42(1.00)$ & $0.92(0.67-1.25)$ & \\
\hline Rest of the world & 73876 & $52(0.70)$ & $0.64(0.49-0.85)$ & 0.004 \\
\hline \multicolumn{5}{|c|}{ Smoking status during pregnancy§ } \\
\hline Did not smoke & 642283 & $648(1.01)$ & 1.00 & \\
\hline Did smoke & 161498 & $198(1.23)$ & $1.22(1.04-1.43)$ & 0.018 \\
\hline \multicolumn{5}{|l|}{$\begin{array}{l}\text { History of orofacial clefts among } \\
\text { offspring }\end{array}$} \\
\hline No & 831214 & $864(1.04)$ & 1.00 & \\
\hline Yes & 1422 & $11(7.74)$ & $7.49(4.12-13.61)$ & $<0.001$ \\
\hline \multicolumn{5}{|l|}{$\begin{array}{l}\text { History of birth defects among } \\
\text { offspring }\end{array}$} \\
\hline No & 795984 & $819(1.03)$ & 1.00 & \\
\hline Yes & 36652 & $56(1.53)$ & $1.37(1.04-1.79)$ & 0.030 \\
\hline \multicolumn{5}{|l|}{ Cleft palate alone } \\
\hline \multicolumn{5}{|l|}{ Year of birth } \\
\hline 1996-1998 & 199863 & $104(0.52)$ & 1.00 & \\
\hline 1999-2001 & 197823 & $84(0.42)$ & $0.82(0.61-1.09)$ & \\
\hline 2002-2004 & 192674 & $88(0.46)$ & $0.88(0.66-1.17)$ & \\
\hline 2005-2008 & 242276 & $81(0.33)$ & $0.64(0.48-0.86)$ & 0.024 \\
\hline \multicolumn{5}{|l|}{$\begin{array}{l}\text { Maternal residence at start } \\
\text { of pregnancyt }\end{array}$} \\
\hline Capital region & 268558 & $91(0.34)$ & 1.00 & \\
\hline Sealand & 106128 & $62(0.58)$ & $1.72(1.25-2.38)$ & \\
\hline Southern Denmark & 172595 & $97(0.56)$ & $1.66(1.25-2.21)$ & \\
\hline Middle Jutland & 190832 & $70(0.37)$ & $1.08(0.79-1.48)$ & \\
\hline Northern Jutland & 82736 & $33(0.40)$ & $1.18(0.79-1.75)$ & $<0.001$ \\
\hline \multicolumn{5}{|c|}{ History of orofacial clefts in offspring } \\
\hline No & 831214 & $352(0.42)$ & 1.00 & $<0.001$ \\
\hline Yes & 1422 & $5(3.52)$ & $8.33(3.44-20.17)$ & \\
\hline \multicolumn{5}{|c|}{$\begin{array}{l}\text { Note: } \mathrm{Cl}=\text { confidence interval, } \mathrm{OR}=\text { odds ratio. } \\
\text { *Likelihood ratio test. } \\
\text { tWomen whose place of residence was not known }(1.4 \%) \text { were excluded from the analysis. } \\
\text { †Women whose country of origin was not known }(0.4 \%) \text { were excluded from the analysis. } \\
\text { §Women for whom smoking status was not known }(3.5 \%) \text { were excluded from the analysis. }\end{array}$} \\
\hline
\end{tabular}


of corticosteroids during the first trimester occurred among 51973 women (6.2\%). During this period, dermatologic corticosteroids were the most common form of the drug used $(43.3 \%)$, followed by other topical forms (23.3\%), nasal sprays (21.6\%), inhalants (14.3\%) and drugs taken orally (4.2\%).

Table 1 shows the pattern of corticosteroid use according to the potential confounders that we assessed.

To identify potential confounders for the association between corticosteroid use and risk of orofacial clefts, we first evaluated the univariable associations between the covariables identified as potential confounders and cleft lip with or without cleft palate and cleft palate alone. Table 2 shows the statistically significant (likelihood ratio test $p<0.05$ ) risk factors for clefts from the univariable analyses.

Women who used any corticosteroid during the first trimester were not significantly more likely to bear offspring with a cleft lip with or without a cleft palate (crude OR 1.05 [95\% CI 0.80-1.37]) or a cleft palate alone (crude OR
1.23 [95\% CI 0.83-1.82]) than women who did not use any corticosteroid during the first trimester. Table 3 shows the adjusted ORs for the association between corticosteroid use during the first trimester of pregnancy and clefts. Testing the homogeneity of effects between any use and all possible forms of use yielded a likelihood ratio $p$ value of 0.3174 for cleft lip with or without cleft palate and a likelihood ratio $p$ value of 0.8907 for cleft palate alone. However, the use of dermatologic corticosteroids was associated with an increased risk of cleft lip with or without cleft palate (OR 1.45 [95\% CI 1.03-2.05]). Corticosteroids in the form of inhalants, nasal sprays and other topicals were not associated with an increased risk of clefts. None of the infants with clefts had been exposed to oral corticosteroids during the first trimester.

Table 4 shows the results of the exploratory analyses of the effects of exposure to dermatologic corticosteroids. Our classification of use according to the daily defined doses during the first trimester and the potency of the corticosteroid did not reveal clear dose-response or potency-response relations.

Table 3: Association between corticosteroid use during first trimester of pregnancy and isolated orofacial clefts among 832636 live births

\begin{tabular}{|c|c|c|c|c|c|}
\hline \multirow[b]{2}{*}{ Corticosteroid use } & \multirow[b]{2}{*}{ No. of live births } & \multicolumn{2}{|c|}{ Cleft lip with or without cleft palate } & \multicolumn{2}{|c|}{ Cleft palate alone } \\
\hline & & $\begin{array}{c}\text { No. } \\
\text { (prevalence*) }\end{array}$ & $\begin{array}{l}\text { Adjusted OR† } \\
\qquad(95 \% \mathrm{Cl})\end{array}$ & $\begin{array}{c}\text { No. } \\
\text { (prevalence*) }\end{array}$ & $\begin{array}{l}\text { Adjusted OR } \\
\qquad(95 \% \mathrm{Cl})\end{array}$ \\
\hline Any use & 51973 & $57(1.10)$ & $1.05(0.80-1.38)$ & $27(0.52)$ & $1.23(0.83-1.82)$ \\
\hline No use & 780663 & $818(1.05)$ & 1.00 & $330(0.42)$ & 1.00 \\
\hline \multicolumn{6}{|l|}{$\begin{array}{l}\text { Route of } \\
\text { administration }\end{array}$} \\
\hline \multicolumn{6}{|l|}{ Oral } \\
\hline Yes & 2195 & $0(0.00)$ & NA & $0(0.00)$ & NA \\
\hline No & 830441 & $875(1.05)$ & 1.00 & $357(0.43)$ & 1.00 \\
\hline \multicolumn{6}{|l|}{ Inhalant } \\
\hline Yes & 7421 & $6(0.81)$ & $0.75(0.34-1.68)$ & $3(0.40)$ & $0.94(0.30-2.92)$ \\
\hline No & 825215 & $869(1.05)$ & 1.00 & $354(0.43)$ & 1.00 \\
\hline \multicolumn{6}{|l|}{ Nasal spray } \\
\hline Yes & 11245 & $6(0.53)$ & $0.52(0.23-1.16)$ & $5(0.44)$ & $1.07(0.44-2.58)$ \\
\hline No & 821391 & $869(1.06)$ & 1.00 & $352(0.43)$ & 1.00 \\
\hline \multicolumn{6}{|l|}{ Dermatologic } \\
\hline Yes & 22480 & $34(1.51)$ & $1.45(1.03-2.05)$ & $14(0.62)$ & $1.45(0.85-2.48)$ \\
\hline No & 810156 & $841(1.04)$ & 1.00 & $343(0.42)$ & 1.00 \\
\hline \multicolumn{6}{|c|}{ Other topical form } \\
\hline Yes & 12091 & $13(1.08)$ & $1.04(0.60-1.79)$ & $5(0.41)$ & $0.97(0.40-2.34)$ \\
\hline No & 820545 & $862(1.05)$ & 1.00 & $352(0.43)$ & 1.00 \\
\hline $\begin{array}{l}\text { Note: } \mathrm{Cl}=\text { confidence } \\
\text { *Per } 1000 \text { live births. } \\
\text { tOdds ratio adjusted } \\
\text { orofacial clefts among } \\
\text { †Odds ratio adjusted }\end{array}$ & $\begin{array}{l}\text { A = not applicable, OF } \\
\text { birth, maternal place } \\
\text { and history of any bir } \\
\text { birth, maternal place }\end{array}$ & $\begin{array}{l}\text { ds ratio. } \\
\text { idence at start o } \\
\text { ects among offs } \\
\text { idence at start o }\end{array}$ & $\begin{array}{l}\text { ncy, maternal place } \\
\text { ncy and history of o }\end{array}$ & $\begin{array}{l}\text { smoking status } \\
\text { efts among offsp }\end{array}$ & egnancy, history of \\
\hline
\end{tabular}


(Note that the daily defined doses for dermatologic corticosteroids were based on the sizes of the packaging and not on the World Health Organization standards, which do not provide such values for creams and ointments.)

We also compared the use of dermatologic corticosteroids during the first trimester of pregnancy with no use of any corticosteroids during pregnancy and with the use of corticosteroids during other periods of pregnancy. The results of the sensitivity analyses support the results of our main analysis and are summarized in Appendix 1 (available at www.cmaj.ca/cgi/content/full /cmaj.101063/DC1). Also included in Appendix

Table 4: Association between use of dermatologic corticosteroid agents during first trimester of pregnancy and isolated orofacial clefts among 832636 live births

\begin{tabular}{|c|c|c|}
\hline & $\begin{array}{l}\text { Cleft lip with or } \\
\text { without cleft palate: } \\
\text { first trimester use }\end{array}$ & $\begin{array}{l}\text { Cleft palate alone: } \\
\text { first trimester use }\end{array}$ \\
\hline $\begin{array}{l}\text { Characteristics } \\
\text { of use }\end{array}$ & $\begin{array}{l}\text { Adjusted OR* } \\
\qquad(95 \% \mathrm{Cl})\end{array}$ & $\begin{array}{c}\text { Adjusted OR } † \\
(95 \% \mathrm{Cl})\end{array}$ \\
\hline
\end{tabular}

\section{Medicationf}

\begin{tabular}{lcclc}
\hline Triamcinolone & 2 & $1.36(0.34-5.47)$ & 0 & NA \\
\hline Hydrocortisone & 3 & $1.28(0.41-3.98)$ & 1 & $1.02(0.14-7.23)$ \\
\hline Betamethasone & 8 & $1.25(0.62-2.51)$ & 6 & $2.30(1.02-5.15)$ \\
\hline $\begin{array}{l}\text { Hydrocortisone } \\
\text { butyrate }\end{array}$ & 15 & $1.82(1.09-3.04)$ & 4 & $1.17(0.43-3.12)$ \\
\hline $\begin{array}{l}\text { Fluocinonide } \\
\begin{array}{l}\text { Mometasone } \\
\text { furoate }\end{array}\end{array}$ & 0 & NA & 1 & $21.27(2.95-153.13)$ \\
$\begin{array}{l}\text { Clobetasol } \\
\text { No. }\end{array}$ & 3 & $0.73(0.23-2.27)$ & 2 & $1.21(0.30-4.86)$ \\
\hline
\end{tabular}

\section{No. of daily}

\section{defined doses}

\begin{tabular}{|c|c|c|c|c|}
\hline $5-20$ & 15 & $1.68(1.01-2.80)$ & 7 & $1.87(0.89-3.97)$ \\
\hline $25-30$ & 17 & $1.98(1.23-3.21)$ & 5 & $1.42(0.59-3.44)$ \\
\hline $35-50$ & 1 & $0.47(0.07-3.31)$ & 1 & $1.13(0.16-8.07)$ \\
\hline$>50$ & 1 & $0.27(0.04-1.90)$ & 1 & $0.66(0.09-4.71)$ \\
\hline \multicolumn{5}{|l|}{ Potency§ } \\
\hline Group I: weak & 3 & $1.28(0.41-3.98)$ & 1 & $1.02(0.14-7.23)$ \\
\hline $\begin{array}{l}\text { Group II: } \\
\text { moderately potent }\end{array}$ & 17 & $1.70(1.05-2.75)$ & 4 & $0.96(0.36-2.57)$ \\
\hline Group III: potent & 11 & $0.99(0.54-1.79)$ & 9 & $2.00(1.03-3.87)$ \\
\hline $\begin{array}{l}\text { Group IV: very } \\
\text { potent }\end{array}$ & 3 & $1.81(0.58-5.63)$ & 0 & NA \\
\hline
\end{tabular}

Note: $\mathrm{Cl}=$ confidence interval, $\mathrm{NA}=$ not applicable, $\mathrm{OR}=$ odds ratio.

*Adjusted for year of birth, maternal place of residence at start of pregnancy, maternal place

of origin, smoking status during pregnancy, history of orofacial clefts among offspring and history of any birth defects among offspring.

tAdjusted for year of birth, maternal place of residence at start of pregnancy and history of orofacial clefts among offspring.

¥Budesonide, fluocortolone, clobetasone, fluoprednidene, alclometasone, hydrocortisone buteprate, desoximetasone, fluocinolone acetonide, diflucortolone, diflorasone, fluticasone, halcinonide and flumetasone were also used, but no orofacial clefts were seen among the offspring of women taking these medications.

$\S$ See Appendix 1 for definition of potency and categorization.
1 are the fully adjusted models including all variables identified as potential confounders, singleton children alone and alternative definitions of the main period of exposure.

\section{Interpretation}

In a large nationwide cohort of live births, general use of corticosteroids during pregnancy was not significantly associated with an increased risk of orofacial clefts. However, the use of dermatologic corticosteroids was associated with an increased risk of cleft lip with or without cleft palate. In contrast, the use of oral corticosteroids, nasal sprays, inhalants or other topical forms was not associated with an increased risk of clefts.

Systemic corticosteroids have been associated with cleft palate and other adverse events in the fetus in rodents. ${ }^{1,25-28}$ Topical corticosteroids are assumed to be safer than systemic corticosteroids. However, corticosteroids have been detected in the fetal blood of some animals after topical application, ${ }^{29}$ and topical use of diflorasone diacetate has been associated with cleft palate in rabbits. ${ }^{30}$

Preparations that contain corticosteroids are among the most frequently prescribed dermatologic treatments. They are commonly used during pregnancy for various skin conditions such as eczema and psoriasis. In humans, topical corticosteroids cross the skin barrier. In a study involving young adults with atopic dermatitis, percutaneous application of $1.0 \%$ hydrocortisone cream yielded median serum cortisol levels of $125 \mathrm{nmol} / \mathrm{L}$ during the acute phase of the condition and $16 \mathrm{nmol} / \mathrm{L}$ during remission. ${ }^{31}$ Furthermore, it has been shown that even for a lowpotency corticosteroid such as hydrocortisone, $15.0 \%$ of the dose crosses the placenta unmetabolized. ${ }^{32}$ These studies suggest fetal serum cortisol levels in the range of $2.4-18.75 \mathrm{nmol} / \mathrm{L}$ after the application of $1.0 \%$ hydrocortisone cream to the mother's skin.

Many previous epidemiologic studies, though not all, ${ }^{7}$ have reported increased risks of orofacial clefts primarily after the use of oral corticosteroids. ${ }^{3-6,8-10}$ However, many of the previous studies were limited by a lack of statistical power. The largest study to date, an American case-control study, included 39 instances of orofacial clefts associated with exposure to corticosteroids, ${ }^{4}$ whereas our study included 84 instances associated with exposure during the first trimester. In addition, many of the previous studies determined corticosteroid use through postnatal interviews, which introduced the potential for recall bias. 
Studies specifically evaluating topical corticosteroids and orofacial clefts are uncommon. ${ }^{46,89}$ A recent Cochrane review of the safety of topical corticosteroids during pregnancy concluded that the current studies were limited and inconclusive and that cohort studies with very large samples were needed. ${ }^{33}$

\section{Limitations}

We relied on national health registers to find infants with orofacial clefts as defined by ICD10 codes. The predictive value of the diagnosis of birth defects in the National Hospital Discharge Registry has previously been evaluated as being high $(88.0 \%) .{ }^{34}$ We would expect these numbers to be higher for clefts alone, and any misclassification would likely bias our results toward no effect.

Our study did not include abortions. This could introduce bias in a study of drug use during pregnancy and the risk of birth defects, if the birth defect itself increases the risk of planned or spontaneous abortion. It is unlikely that such a bias played a role in a study of birth defects such as isolated orofacial clefts.

We relied on a national prescription drug registry to determine corticosteroid use with the assumption that a filled prescription would lead to use of the drug. However, some divergence with respect to use and timing of use is to be expected, and this divergence would bias our results toward no effect. We were not able to include information on over-the-counter use or hospital use of corticosteroids in this study. Again, we expect that any misclassification would bias our results toward no effect.

Confounding by indication is not obvious in our study; the indications for corticosteroid use are many, and none has been associated with orofacial clefts. That no association was seen between corticosteroid use during late pregnancy and risk of orofacial clefts, despite the apparent increase in risk seen with the use of dermatologic corticosteroids during early pregnancy, also supports the minimal impact of confounding by indication.

The absence of risk associated with corticosteroids taken orally or as inhalants seen in our study should be evaluated in the context of the study's statistical power, which was limited, particularly for oral forms of the drug. However, moderate to high risks can be excluded. For example, for inhalants, we can exclude an increase in the risk of cleft lip with or without cleft palate that is higher than $68 \%$.

The observed association between dermatologic corticosteroids and orofacial clefts in our study may be a result of multiple statistical comparisons. Given that exploratory analyses of the dose-response and potency-response relations between the use of dermatologic corticosteroids and cleft lip with or without cleft palate did not support a causal association, we cannot exclude that the observed results are non-causal random effects. The overall association between dermatologic corticosteroids and orofacial clefts appeared to be carried by hydrocortisone butyrate alone, a corticosteroid on the lower end of the potency scale.

\section{Conclusion}

Our results add to the safety information for a class of drugs commonly used during pregnancy. Our study did not show an adverse effect of corticosteroid use during pregnancy on the risk of orofacial clefts. However, the absence of risk associated with corticosteroids taken orally or as inhalants seen in our study does not necessarily show that these products are safe for use during pregnancy.

If the observed association between dermatologic corticosteroids and orofacial clefts seen in our study is causal, it is in contrast to the lack of association seen for corticosteroids taken orally or as inhalants. Since indepth investigation of the pattern of association between orofacial clefts and use of dermatologic corticosteroids during pregnancy indicated that this result did not signify a causal connection, it is likely that this association arose from multiple statistical comparisons.

\section{References}

1. Fraser FC. Fainstat TD. Production of congenital defects in the off-spring of pregnant mice treated with cortisone; progress report. Pediatrics 1951;8:527-33.

2. Fraser FC, Sajoo A. Teratogenic potential of corticosteroids in humans. Teratology 1995;51:45-6.

3. Carmichael SL, Shaw GM. Maternal corticosteroid use and risk of selected congenital anomalies. Am J Med Genet 1999;86:242-4.

4. Carmichael SL, Shaw GM, Ma C, et al. Maternal corticosteroid use and orofacial clefts. Am J Obstet Gynecol 2007;197:585-7.

5. Czeizel AE, Rockenbauer M. Population-based case-control study of teratogenic potential of corticosteroids. Teratology 1997; 56:335-40.

6. Edwards MJ, Agho K, Attia J, et al. Case-control study of cleft lip or palate after maternal use of topical corticosteroids during pregnancy. Am J Med Genet A 2003;120A:459-63.

7. Källén B, Rydhstroem H, Aberg A. Congenital malformations after the use of inhaled budesonide in early pregnancy. Obstet Gynecol 1999;93:392-5.

8. Källén B. Maternal drug use and infant cleft lip/palate with special reference to corticoids. Cleft Palate Craniofac J 2003;40:624-8.

9. Pradat P, Robert-Gnansia E, Di Tanna GL, et al. First trimester exposure to corticosteroids and oral clefts. Birth Defects Res A Clin Mol Teratol 2003;67:968-70.

10. Rodríguez-Pinilla E, Martinez-Frias ML. Corticosteroids during pregnancy and oral clefts: a case-control study. Teratology 1998;58:2-5

11. Knudsen LB, Olsen J. The Danish Medical Birth Registry. Dan Med Bull 1998;45:320-3.

12. Jørgensen FS. Ultrasonography of pregnant women in Denmark 1999-2000. Description of the development since 1980-1990 [article in Danish]. Ugeskr Laeger 2003;165:4409-15.

13. Andersen TF, Madsen M, Jorgensen J, et al. The Danish National Hospital Register. A valuable source of data for modern health sciences. Dan Med Bull 1999;46:263-8. 
14. Bille C, Olsen J, Vach W, et al. Oral clefts and life style factors - a case-cohort study based on prospective Danish data. Eur J Epidemiol 2007;22:173-81.

15. Erickson JD. Risk factors for birth defects: data from the Atlanta birth defects case-control study. Teratology 1991;43:41-51.

16. Leite IC, Paumgartten FJ, Koifman S. Chemical exposure during pregnancy and oral clefts in newborns. Cad Saude Publica 2002; 18:17-31.

17. Métneki J, Puho E, Czeizel AE. Maternal diseases and isolated orofacial clefts in Hungary. Birth Defects Res A Clin Mol Teratol 2005;73:617-23.

18. Mossey P. Epidemiology underpinning research in the aetiology of orofacial clefts. Orthod Craniofac Res 2007;10:114-20.

19. Puhó EH, Szunyogh M, Metneki J, et al. Drug treatment during pregnancy and isolated orofacial clefts in hungary. Cleft Palate Craniofac J 2007;44:194-202.

20. Robert E, Kallen B, Harris J. The epidemiology of orofacial clefts. 1. Some general epidemiological characteristics. J Craniofac Genet Dev Biol 1996;16:234-41.

21. Saxén I. Associations between oral clefts and drugs taken during pregnancy. Int J Epidemiol 1975;4:37-44.

22. Wyszynski DF, Beaty TH, Maestri NE. Genetics of nonsyndromic oral clefts revisited. Cleft Palate Craniofac J 1996;33:406-17.

23. Yang J, Carmichael SL, Canfield M, et al. Socioeconomic status in relation to selected birth defects in a large multicentered US case-control study. Am J Epidemiol 2008;167:145-54.

24. Pedersen CB, Gotzsche H, Moller JO, et al. The Danish Civil Registration System. A cohort of eight million persons. Dan Med Bull 2006;53:441-9.

25. Nanda R, van der Linden FP, Jansen HW. Production of cleft palate with dexamethasone and hypervitaminosis A in rat embryos. Experientia 1970;26:1111-2.

26. Nasjleti CE, Avery JK, Spencer HH, et al. Tritiated cortisone distribution and induced cleft palate in mice. J Oral Ther Pharmacol 1967;4:71-82.

27. Shah RM, Kilistoff A. Cleft palate induction in hamster fetuses by glucocorticoid hormones and their synthetic analogues. J Embryol Exp Morphol 1976;36:101-8.

28. Walker BE. Induction of cleft palate in rabbits by several glucocorticoids. Proc Soc Exp Biol Med 1967;125:1281-4.
29. Yamada H., Nakano M., Ichihashi T. Fetal concentration after topical application of betamethasone 17,21-dipropionate (S-3440) ointment and teratogenesis in mice and rabbits. Pharmacometrics 1981;21:645-55

30. Narama I. Reproduction studies of diflorasone diacetate (DDA) (4) Teratogenicity study in rabbits by percutaneous administraton. Pharmacometrics 1984;28:241-50.

31. Turpeinen M, Mashkilleyson N, Bjorksten F, et al. Percutaneous absorption of hydrocortisone during exacerbation and remission of atopic dermatitis in adults. Acta Derm Venereol 1988;68:331-5.

32. Murphy BE, Clark SJ, Donald IR, et al. Conversion of maternal cortisol to cortisone during placental transfer to the human fetus. Am J Obstet Gynecol 1974;118:538-41.

33. Chi CC, Lee CW, Wojnarowska F, et al. Safety of topical corticosteroids in pregnancy [review]. Cochrane Database Syst Rev 2009;(3):CD007346.

34. Larsen H, Nielsen GL, Bendsen J, et al. Predictive value and completeness of the registration of congenital abnormalities in three Danish population-based registries. Scand J Public Health 2003;31:12-6.

Affiliation: From the Department of Epidemiology Research, Statens Serum Institut, Copenhagen, Denmark

Contributors: Anders Hviid had full access to all data in the study and guarantees the integrity of the data and accuracy of the analyses. Anders Hviid drafted the manuscript. Ditte MølgaardNielsen revised the manuscript critically for important intellectual content. Both authors have contributed substantially to the design, acquisition of data, analysis and interpretation, and have approved the final version submitted for publication.

Funding: The study was supported by the Danish Medical Research Council and the Lundbeck Foundation. The funding agencies had no role in the design and conduct of the study; nor in the collection, management, analysis and interpretation of the data; nor in the preparation, review and approval of the manuscript.

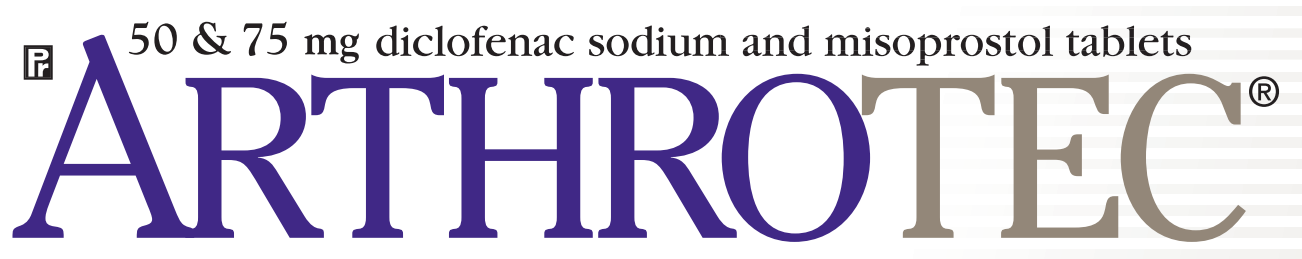

An NSAID with a mucosal protective agent, PrARTHROTEC ${ }^{\circledR}$ (50 \& 75 mg diclofenac sodium and misoprostol tablets) has contraindications as well as warnings and precautions of use.

Please consult the Prescribing Information, which is available on the ARTHROTECMD.ca website, or the Product Monograph, which is available upon request.

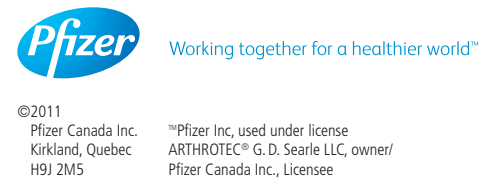

